\title{
Development of a Software to Plan UAVs Stereoscopic Flight: An Application on Post Earthquake Scenario in L'Aquila City
}

\author{
Valerio Baiocchi ${ }^{1}$, Donatella Dominici ${ }^{2}$, Maria Vittoria Milone ${ }^{1}$, \\ and Martina Mormile ${ }^{1}$ \\ 1 "La Sapienza" University- DICEA, Rome, Italy \\ \{valerio.baiocchi, mariavittoria.milone, \\ martina.mormile\} auniroma1.it \\ ${ }^{2}$ Università dell'Aquila, Dipartimento Ingegneria Civile, Edile-Architettura, \\ Ambientale (DICEAA), Via Gronchi 18 \\ donatella.dominici@univaq.it
}

\begin{abstract}
On April 6, 2009, an earthquake hit the historic center of L'Aquila city, hundreds of victims, thousands of collapses. During the post-emergency a continuous monitoring of all building is crucial in order to guarantee that each structure at least will not worsen its stability until the final reconstruction is completed. So detailed surveying of all building is performed using different geomatic techniques as total stations, land photogrammetry, and laser scanners. Even if all these techniques can perfectly respond to many crucial post hazard needs, there are still many monitoring that cannot be completely carried on with traditional techniques. For these reasons, in this work, the advantages of using multirotor UAVs will be illustrated; UAVs can be fully remote controlled and so the geometry of photogrammetric image acquisition can be imposed.

For this task planning of flight is essential so a package was realized to obtain actual photogrammetric stereoscopic acquisitions.
\end{abstract}

Keywords: UAV, earthquake, flight planning, L'Aquila.

\section{Introduction}

The historic center of the city of L'Aquila suffered big damages from the main seismic event of 6th April 2009. The main shock occurred at 3:32 local time (1:32 UTC) on 6 April 2009, and was rated 5.8 Richter magnitude and 6.3 on the moment magnitude; its epicenter was near L'Aquila, the capital of Abruzzo, which together with surrounding villages suffered most damage. There have been several thousand foreshocks and aftershocks since December 2008, more than thirty of which had a Richter magnitude greater than 3.5. The earthquake was felt throughout central Italy; 297 people are known to have died, making this the deadliest earthquake to hit Italy since the 1980 Irpinia (southern Italy) earthquake. Overall, the region affected by the seismic activity covers an ellipse-shaped area measuring about $15 \mathrm{~km}$ in length, parallel to the Apennines mountain chain and $5 \mathrm{~km}$ in wideness. According to the 
Italian National Institute for Geophysic and Vulcanology (INGV) this seismic activity is the result of a normal fault movement on a NW-SE oriented structure which is part of the $800 \mathrm{~km}$ long segmented vertical fault system running all along the Apennines mountains and covering great number of Italy's most important monumental cities. Within this urban setting, the seismic event caused serious damage to numerous important buildings, mainly including a valuable heritage that goes from the Baroque and Renaissance periods, including important churches and palaces. All the masonry buildings, including the traditional palaces and high-density residential quarters in the old city centre, suffered severe damage and many partial collapses. Many of these buildings survived anyway, their general collapse being in most cases avoided by tie, rods, anchor plates and other simple but effective earthquake-resistant presidium suggested by the historical seismic nature of the land. During the weeks immediately after the main event fire department and civil protection teams came to L'Aquila from all over Italy to make safe as many damaged buildings as it was possible. The first emergency stage took months to be completed and after it was evident that only a small step of the whole operation was done. Certainly, the first thing to do in these situations is a detailed documentation of all structures in order to gather all the vital information for a correct planning of the further operations. Moreover, during the emergency a continuous monitoring of all building is crucial in order to guarantee that each structure at least will not worsen until the final reconstruction is completed.

To do so, detailed surveying of all building is performed using geomatic instruments as total stations, close range photogrammetry, laser scanners and deformation monitoring. Even if all these techniques can perfectly fulfill many crucial post hazard needs, there are still many cases where they show some limits. Most of the difficulties are related to the morphologic and architectural accessibility, problems typical of a post disaster scenario. During a post-seismic period, surveying with classical topographic instruments is not only difficult but also complex because it has to be planned also considering the possible danger in the area. Other methodologies like aerial photogrammetry are surely less dangerous but would not in most case produce detailed and accurate information for the damaged structures.

On the other hand, using micro UAVs (Unmanned Aerial Vehicles) for surveying in such particular cases can easily bypass many of these problems [1] [2]. For example there is no accessibility problems for an UAV for the flexible flying capabilities of these instruments that can virtually permit the access of any place. The security for these instruments that are remotely controlled reduce the risks for the operator because he can be far from the area to be surveyed.

\section{$2 \quad$ Flight Planning}

The flight planning is a rather complex phase because it influences deeply the results [3] [4] [5]. A correct photogrammetric survey, in fact, needs a suitable number of high quality acquisitions with the correct geometry [6] [7] [8]. To guarantee this quality the whole flight and thus the acquisition points have to be spatially distributed maintaining definite geometric characteristics as equal and correct air base and correct interaxes [9]. Considering as a guideline, the case of a traditional photogrammetric 
airplane acquisition, a flight is planned according to predetermined almost rectilinear paths parallel to each other; during the flight, images are taken in succession at regular time intervals, forming what is called a "swath". To determine the density and the number of the needed waypoints some metric information are necessary. Firstly the flying altitude and the flying speed to simulate the temporal intervals needed to cover all distances between each couple of waypoints.

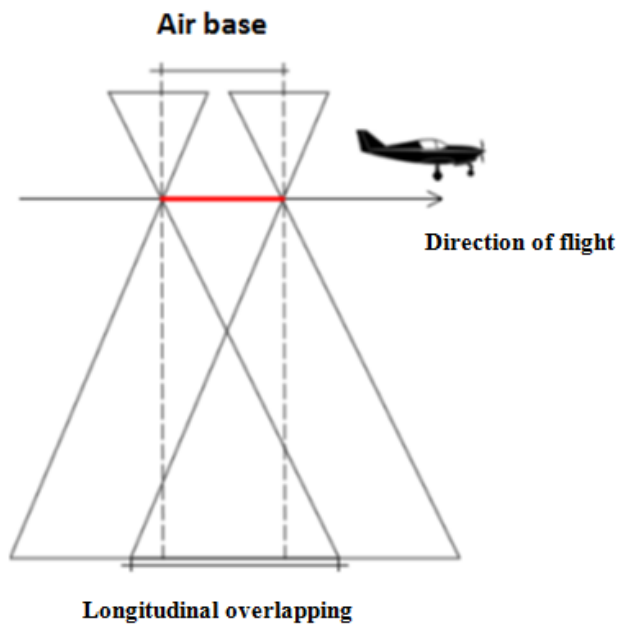

Fig. 1. Case of a traditional photogrammetric flight

Then, the geometric characteristics of the camera also known as "internal orientation of the camera" have to be found: the focal length, pixel dimensions and the frame format in order to correctly determine the physical area covered by each acquisition. Obtained all these information and using a flight planning software each waypoint is calculated in order to guarantee a minimum of $60 \%$ overlap between each couple of frames in the longitudinal direction ensuring in this way also a stereoscopic vision and thus the three-dimensionality of all objects in the model that will be recreated (Fig. 1). In addition to these parameters, another characteristic for the planning is the expected average scale of the photogram. This scale expresses the relation between the dimension of an element in the photogram and the actual dimension of the element. Due to the fact that the terrain usually has not a flat morphology, the distance between each pixel and the acquisition point is not the same, thus an average value of this distance is used instead to evaluate the average scale. The average scale is a guideline to correctly generate maps at the required scale. All this is correct for metric cameras on photogrammetric flight; we want to investigate if this approach is correct also for the multirotor UAVs using amateur or semi-metric cameras[10] [11]. The scope is to obtain all and only the frames needed to reconstruct the stereoscopy and to obtain geometrically correct reconstruction of the objects observed to reconstruct a DSMs [12] [13]. 
The optimization of flight plans is also of strategic importance considering, on one hand, the current reduced autonomy of some UAV, on the other hand the interest in possible expansion of the applications to the production of complete cartography of some areas. Moreover a scheduled flight would help multitemporal investigations as, for example, a repeated flight, with identical parameters, may allow to immediately detect changes in the areas due to unexpected intrusion of other subjects, sudden changes of light, etc.

\subsection{The UP23 Package}

The software "UP23" (UAVs planner to 3 dimensional acquisitions) developed requires as input data the size and shape of the area to be acquired, the optic parameters of the camera installed on the drone and the desired final scale of the acquisitions. From these first input data the package suggest a flight altitude, which is proposed to the user which is free to edit if it's not suitable for the area to be investigated (for example, there is an obstacle with a height near to the one estimated).

Once estimated the flight height and consequently the final scale obtainable, the values of longitudinal and across overlap have to be inserted in the software to allow the evaluation of the airbase needed to assure a correct stereoscopic reconstruction.

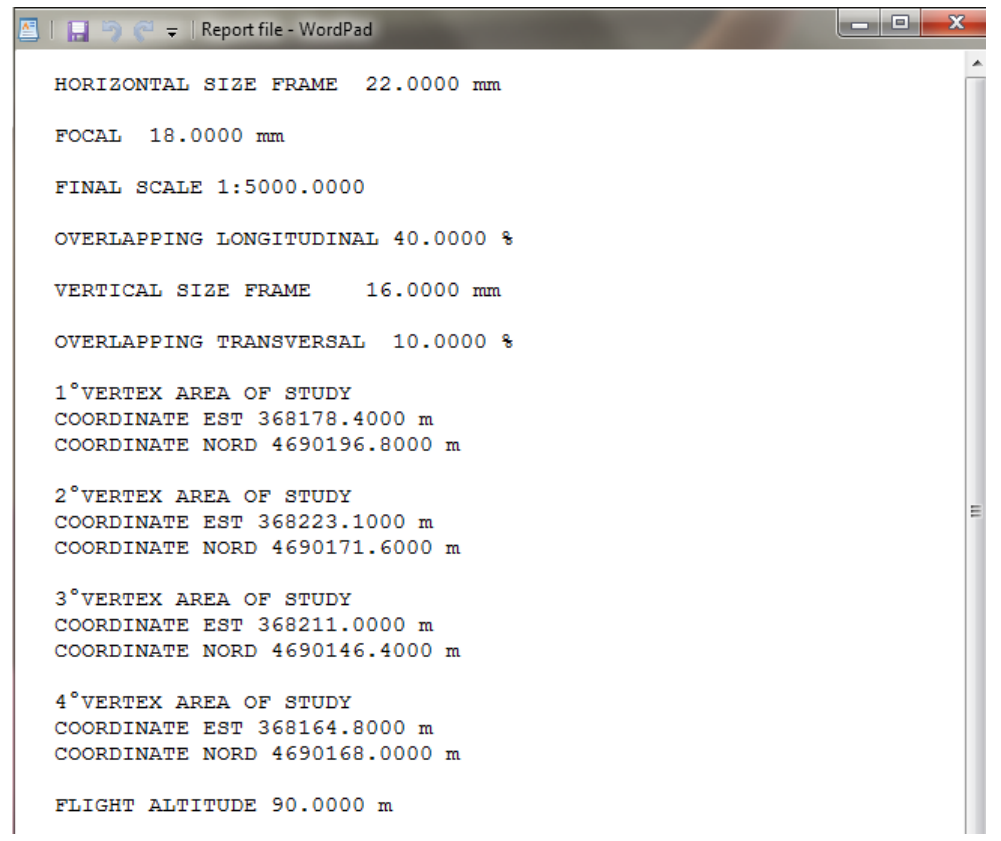

Fig. 2. Report file obtained after processing software UP23 
Then the software proceeds to the calculation of "waypoints" and correspondent footprint of each acquisition. As output the software returns the coordinates of the points where the UAVs has to stop and acquire the images; also a vector files of polygons that shows footprint of each frame is produced.

On completion of the program, it provides a report file, which also contains the intermediate results that were estimated during its execution. This file is shown in figure 2.

In the diagram shown in the Fig. 3. we can see how the software works.

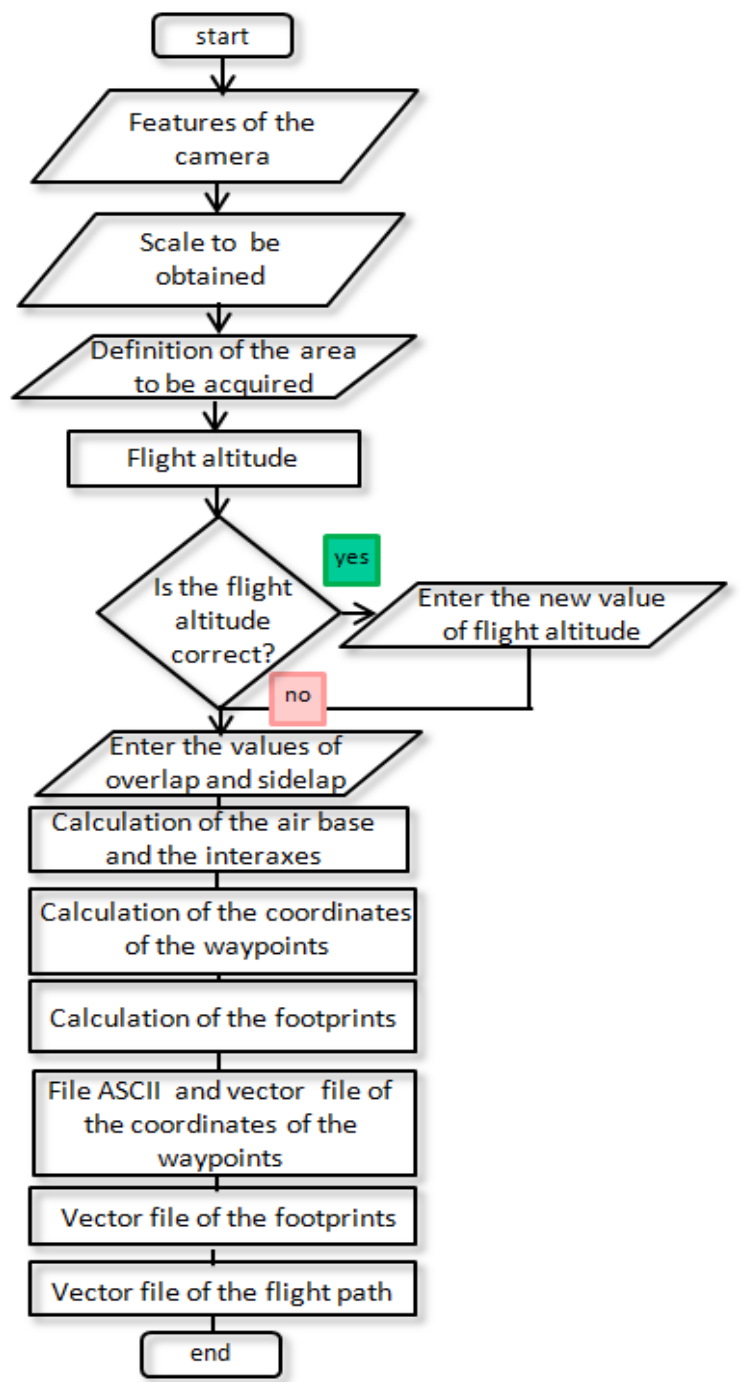

Fig. 3. Flow chart of the software UP23 
Vector files can then be loaded into a GIS environment as the open source Q-Gis that allow to overlap to public access orthophoto satellite maps or to existing raster or vector cartography as shown in Fig. 4.

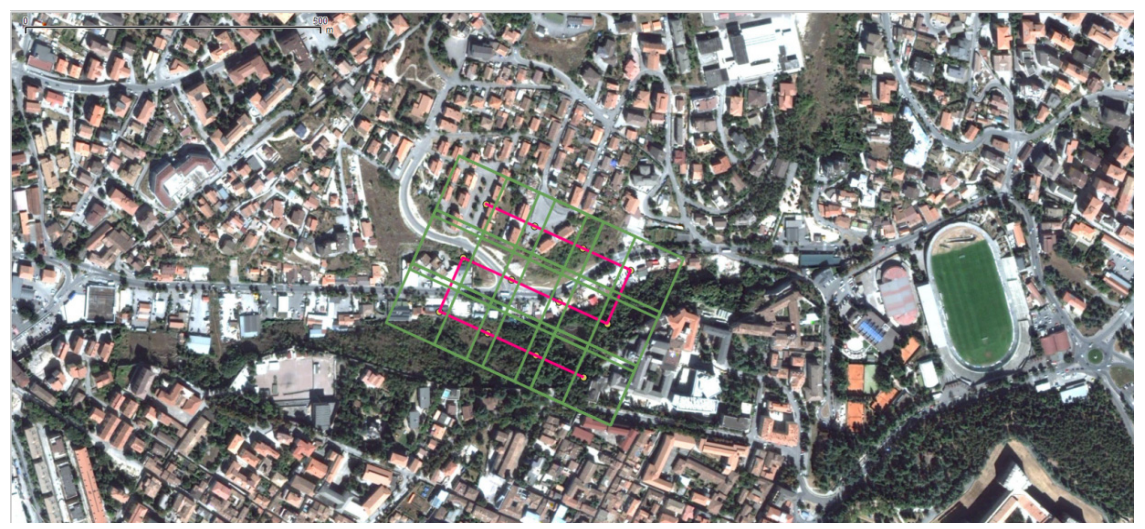

Fig. 4. Vector files loaded into the open source software QGIS

\section{Case Study}

To realize this experiment a typical italian square with historic-monumental buildings was chosen: "Piazza Palazzo" in L'Aquila city (Fig. 5). Its dimensions are approximately 60 meters long to 38 meters wide covering a total area of 2500 square meters and surrounded by the public library, the city hall "Palazzo Margherita" with its $40 \mathrm{~m}$ height bell tower.

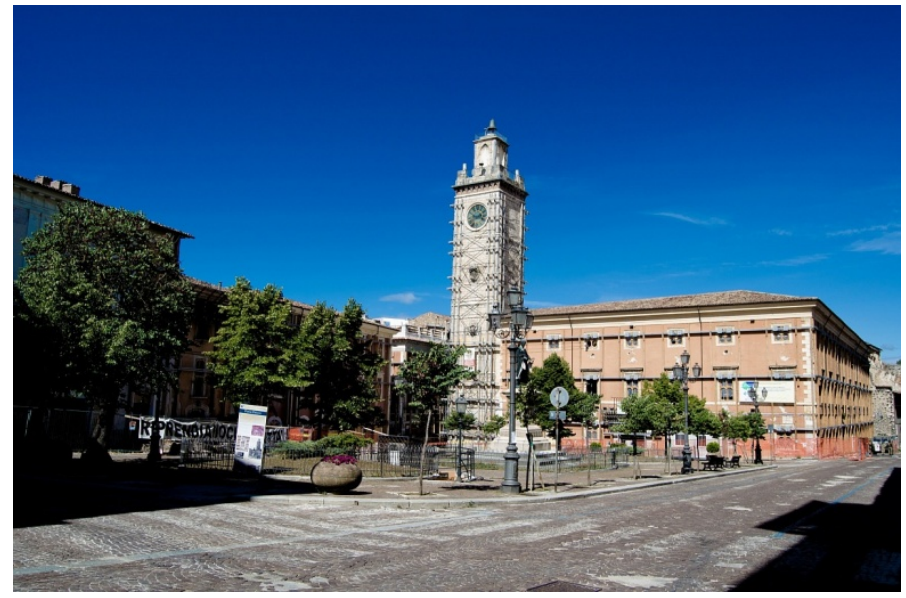

Fig. 5. The test area: Piazza Palazzo 
This experiment in the historic center of L'Aquila for this project is the result of a collaboration between the Faculties of Engineering of L'Aquila and Rome university, and the IPT company of Rome, that gently provided a Mikrokopter platform in Okto configuration, including all on board sensors like Reflex camera and GNSS navigation receivers to enable the automatic flight mode also with the necessary transmitters able to send all telemetry data in real time to the ground control station. The choice of this platform was made trying to achieve excellent flying characteristics that can allow an optimum stability even in presence of wind or other non optimal climatic conditions and according to the payload specified by the manufacturer, which allows carrying a reflex digital camera. In particular, a Canon EOS 550D equipped with a wireless video transmission to the ground was chosen to allow a better control of the flight and acquisitions in real time.

\section{Comparison of Automated Planning with "Classical" Planning}

Once the UAV takes off, the mode of autonomous flight can be activated; in such modality were acquired 15 frames all over the square during the "manual" acquisition tested. The entire route taken by the drone was continuously monitored using wireless video transmission on this platform, so to control on the monitor shown in the Fig. 6. the sequence of shots made by the camera of each waypoint planned manually by the operators; in the figure 7 is shown a frame acquired in this modality.

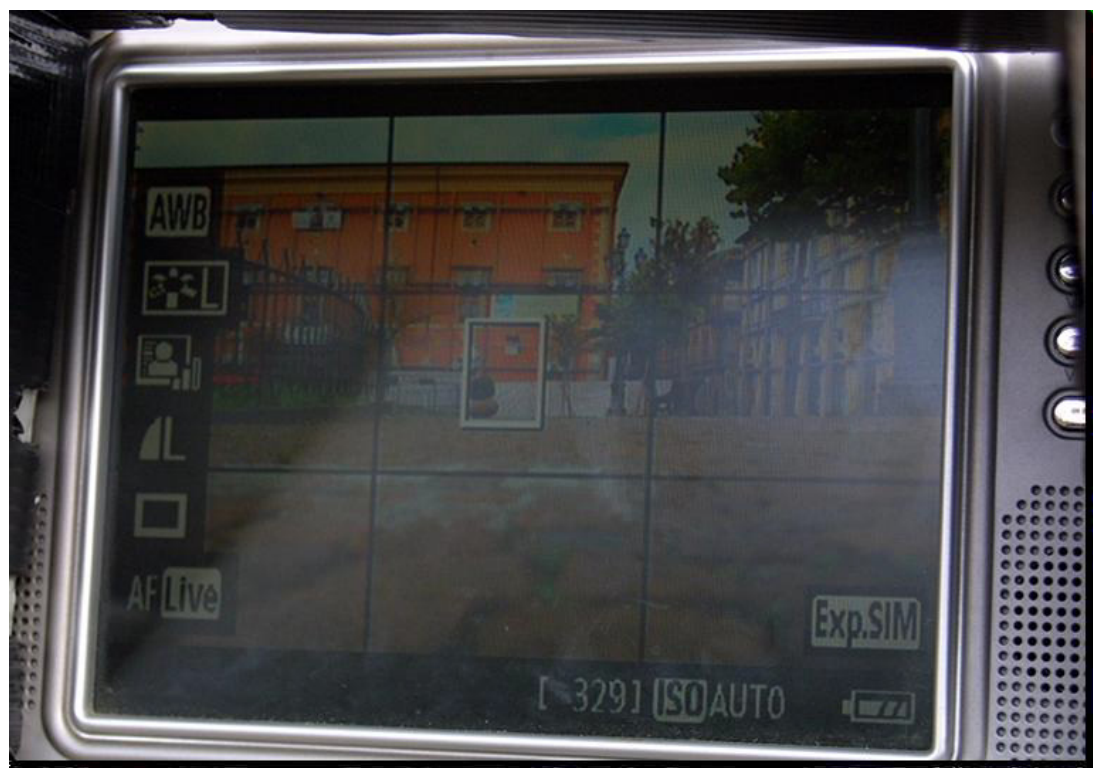

Fig. 6. Monitor connected to wi fi with reflex camera 


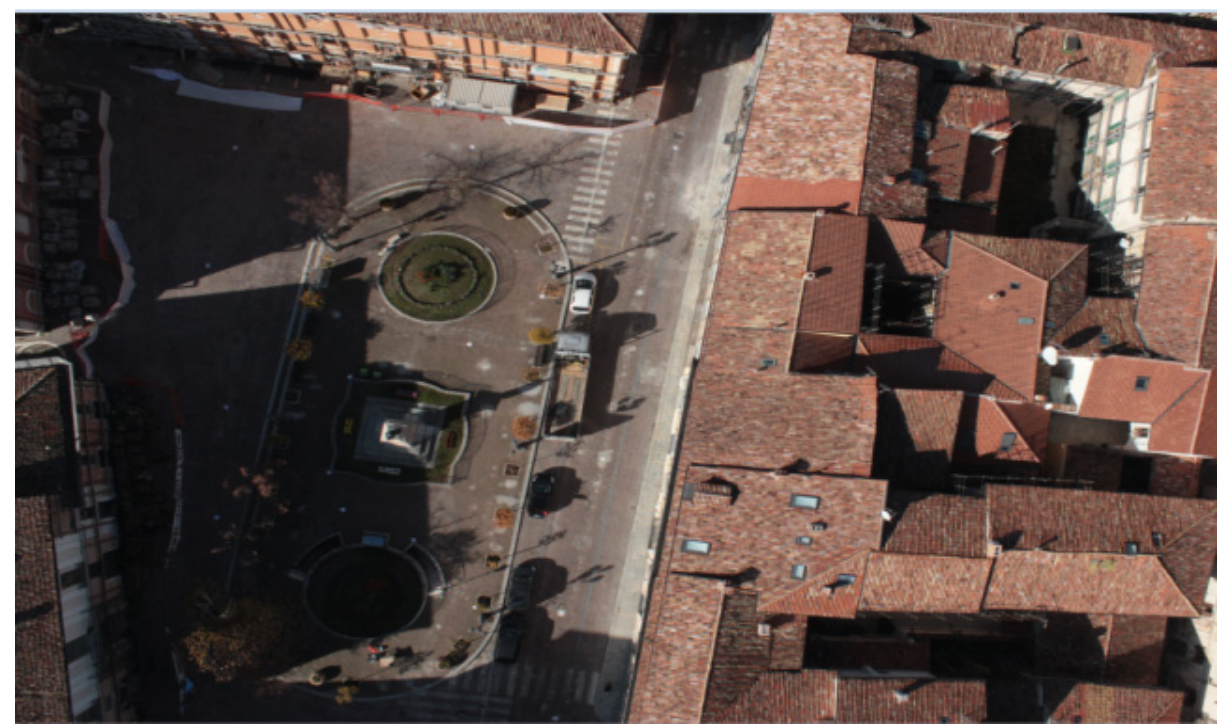

Fig. 7. Frame acquired through a flight planning manual

In addition to the frames, was also recorded the entire GPS track of flight on a Micro SD memory card on the back of the Navi-Ctrl platform. An extract from the XML file, which shows how the track is stored at each point is shown below:

$<$ ?xml version="1.0" encoding="UTF-8" standalone="no" ?> $<$ gpx creator="NC" version="2.0" >

$<$ metadata $>$

<link href="http: / / www.mikrokopter.de" >

$<$ text $>$ MikroKopter</text $>$

$</$ link $>$

$<$ desc $>$ FC HW:2.1 SW:0.86d + NC HW:1.1 SW:0.26f</desc $>$

$</$ metadata $>$

$<$ trk $>$

$<$ name $>$ Flight $</$ name $>$

$<$ trkseg>

<trkpt lat $="+42.3510661 "$ lon $="+13.3986718 ">$

$<$ ele $>80.563</$ ele $>$

<time>2011-11-15T11:01:39z</time>

$<$ sat $>10</$ sat $>$

$<$ extensions $>$

$<$ Altimeter $>1601$, ' = '</Altimeter $>$

$<$ Variometer $>-3</$ Variometer $>$

$<$ Course $>132</$ Course $>$

$<$ GroundSpeed $>12</$ GroundSpeed $>$

$<$ Verticalspeed>-11</Verticalspeed>

<FlightTime>208</FlightTime> 
$<$ Voltage $>14.7</$ Voltage $>$

$<$ Current $>34.8</$ Current $>$

$<$ Capacity $>1910</$ Capacity $>$

For each point, the navigation system stores a set of information including: geographical coordinates in degree of the plotted point, flight altitude, aircraft speed, number of satellites tracked, distance from the waypoint to reach, plus a set of additional data of telemetry regarding the status of battery or engine operation.

Thanks to this XML file it was possible to reconstruct the path performed by the drone assisted by the pilot, the points where the drone remained more time were identified as waypoints. We can see in figure 8 the trajectory performed by the UAV during the manual operated flight.

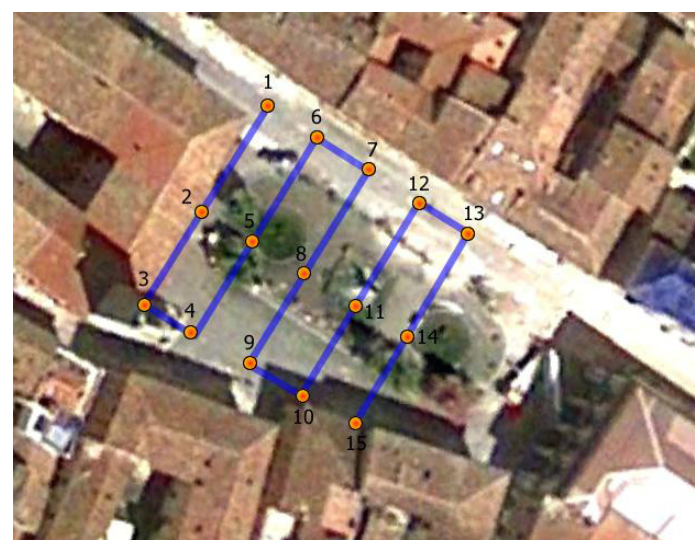

Fig. 8. Trajectory performed following a manually planned flight at 80 meters flight altitude

As previously noted, 15 acquisitions over an area of 60x40 meters extension are numerous, especially when you consider the limited autonomy of the micro UAVs.

UAV pilot, on his experience, decided to flight two times: the first with an height of 60 meters with full cover of the square and the second at 80 meters but only with some spot acquisitions because the drone showed a little instability, due probably to particular meteorological conditions. For the first flight the pilot covered the area with 15 acquisitions while our software estimated only 6 acquisitions. For the second test at 80 meters our software evaluated that only two shoot were needed. We would like to underline that this is only a first experimentation and these results have certainly to be verified, because it has not yet been verified if the planned six shots can actually generate a stereoscopic coverage.

In figure 9 and figure 10 we can see the acquisitions planned by the software for the two different flight height; comparing figure 8 with figure 10 we can observe the drastic reduction of images needed. 


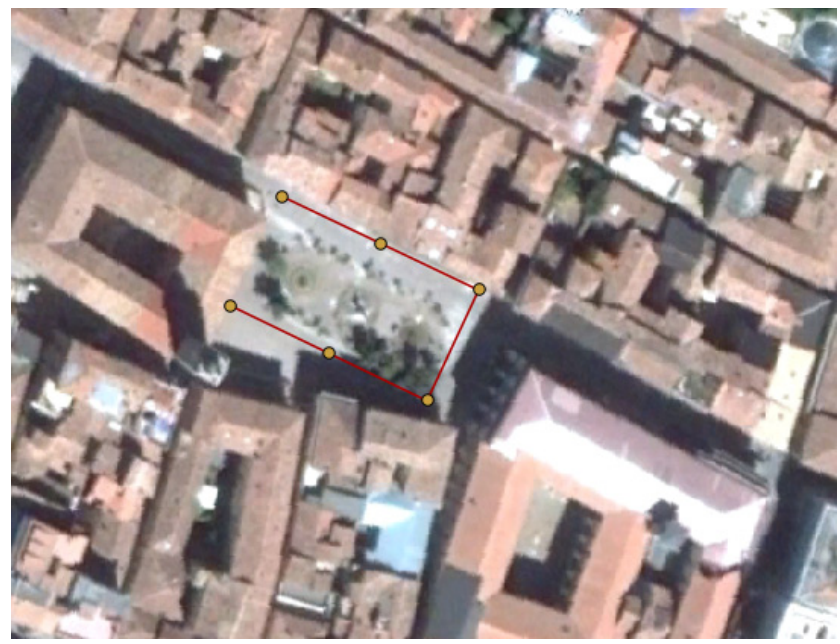

Fig. 9. Trajectory planned by the software UP 23 considering a flight altitude of 60 meters, $60 \%$ overlapping longitudinal, $20 \%$ overlapping transverse, air base of 70 meters and interaxes 45 meters

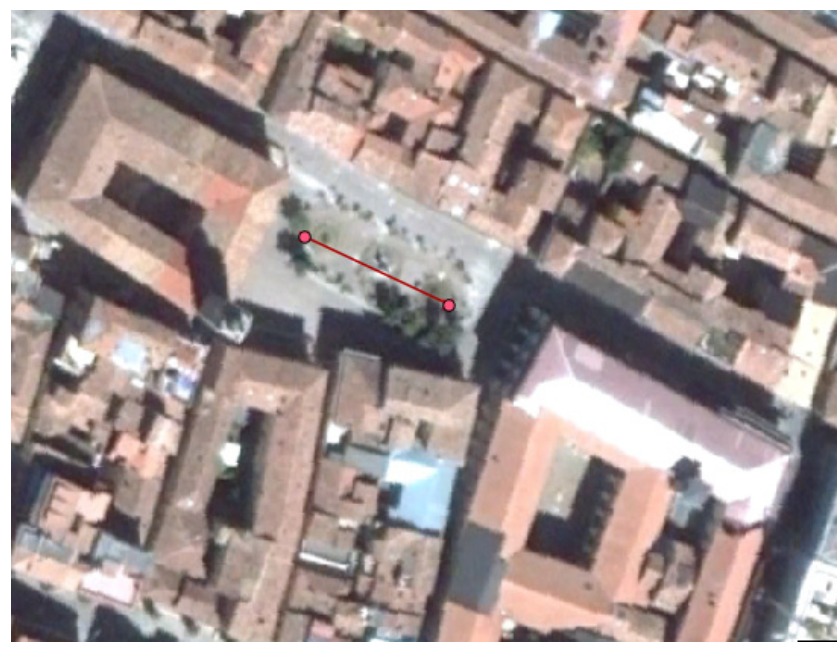

Fig. 10. Trajectory planned by the software UP23 considering a flight altitude of 80 meters, $60 \%$ overlapping longitudinal, $20 \%$ overlapping transverse, air base of 40 meters and interaxes 54 meters

The first tests on the DSM automatic extraction confirmed that the number of acquisitions executed by the pilot was extremely redundant, in fact the baseline between consecutive acquisitions resulted too short and so approximately 7 acquisitions were useless for DSM extraction. 


\section{$5 \quad$ Results}

Considering the simulation performed with the software to a flight altitude of 80 meters, from the two acquisitions was extracted a DSM through the use of software PCI Geomatica 2012.

The treatment of the information content of aerial images acquired with the UAV the extraction of their metric characteristics requires some preliminary, such as camera calibration. In this case, the camera calibration has been realized through the use of software Photomodeler, which allowed to obtain the parameters which enable to the mathematical model to calculate the internal orientation and then to identify the relationship between camera and frame (Fig.11).

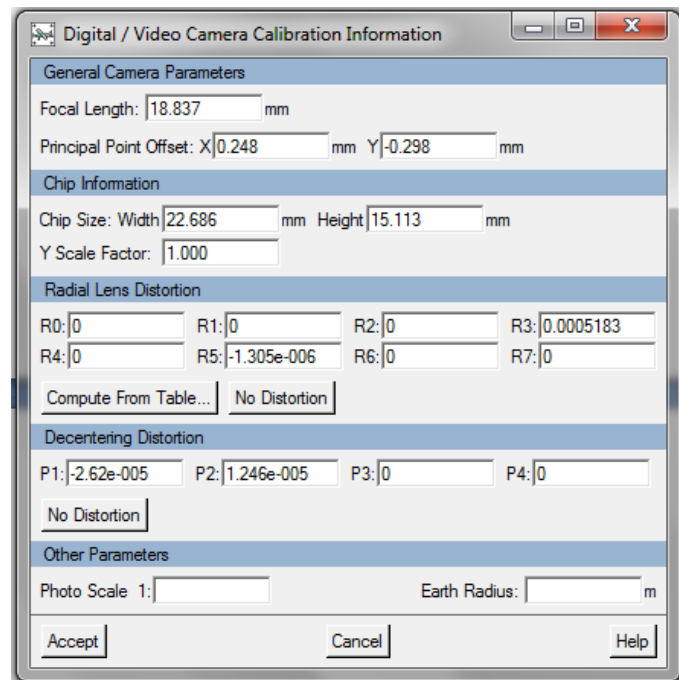

Fig. 11. Screen for entering the calibration parameters of the camera

The extraction of a DEM from a photogrammetric survey need at least a pair of frames, with a minimum overlap of $60 \%$, as the stereoscopy required to $3 \mathrm{D}$ relief is realized only in this area of overlap. In this case, we have considered the two acquisitions between those made by the pilot UAVs, that come closest to those indicated by the software UP23. In the present case, the frames which form what is called a stereo pair, are chosen with a distance between the air base equal to approximately $39 \mathrm{~m}$ and consequently with an overlap of $62 \%$ (Fig.12).

To take advantage of stereoscopic vision for the extraction of DSM is necessary to reconstruct the geometry of the two images in relation to the surface, calculating position and orientation of the camera relative to the ground in moment in which the frames were acquired. 


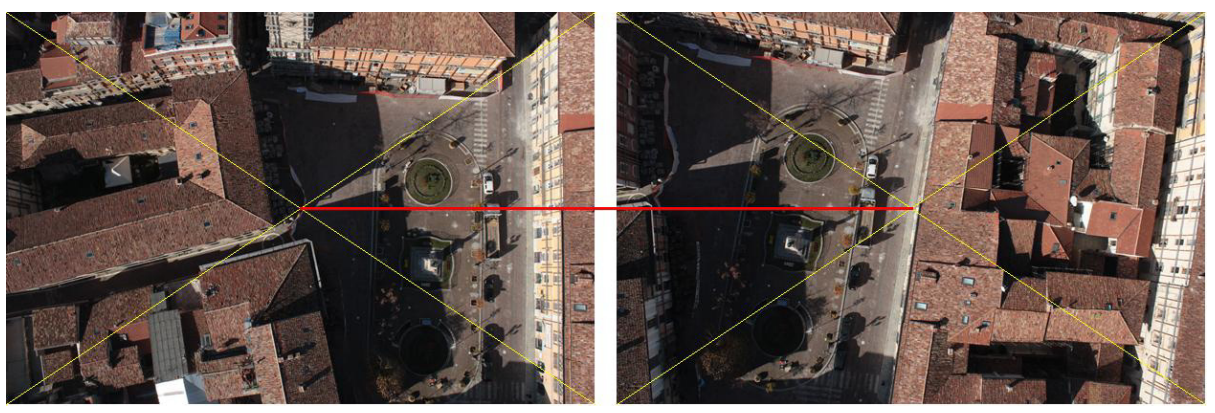

Fig. 12. Stereo pair used for the extraction of DSM on Piazza Palazzo (is highlighted by red segment in the distance between the air bases)

This operation, called "absolute orientation", is also part of external orientation and is performed starting from the identification of each frame of the Ground Control Point (GCP) and placing in the software of coordinates of the same. In the case of Piazza Palazzo, which extends for about $60 \times 38 \mathrm{~m}$, we chose instead to detect in total 21 control points, corresponding in part to the masonry elements or edges already present in the square, and in part to points arranged on the road, along the perimeter. The points have been acquired by a receiver Precision GNSS RTK (Real Time Kinematic). After entering the GCP is necessary to orient the two frames in order to reassemble the mutual position when shooting, this operation, called "relative orientation", is part of the process of external guidance and you make it through the identification of Tie Points.

After the placing of the points, have been created epipolar images, which are shown in Figure 13, where it was also drawn a hypothetical axis along which are the homologous points.
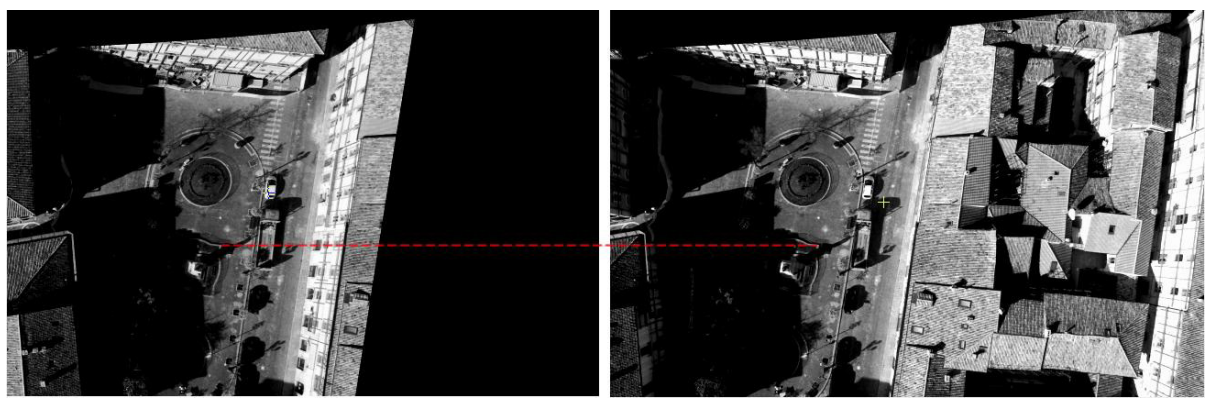

Fig. 13. Epipolar images generated from stereo pair choice between frames captured by the drone 


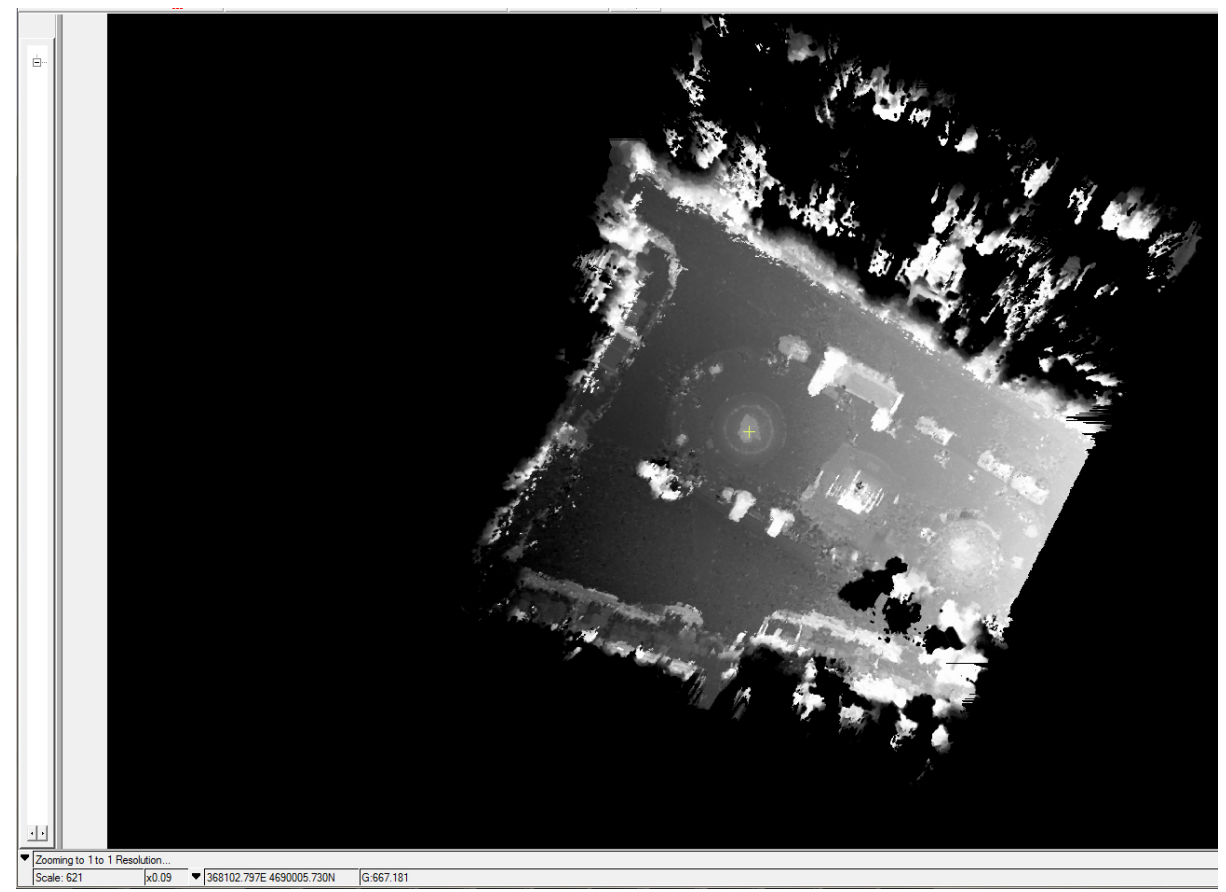

Fig. 14. DSM resulting from the process of automatic extraction

The DEM obtained is shown in figure 14;

In figure 14, we can observe that an impressive precision characterize the obtained DSM, in fact object with little elevation from the ground are visible: note the circular boardwalk in the centre of the image, that is only 10 centimeter high from the ground.

On the other hand, considering the accuracy only some very initial tests was possible to perform. Presently, in fact, only a little number of spot points extracted from medium-high scale digital cartography are available to check DSM elevations. From these introductory validation a maximum difference of around one meter was observed, and this value is contained in the expected error for the cartography at this scale. This test only hints that the obtained DSM is more accurate than the cartography, but more has to be performed with a larger number of points with an higher accuracy: for this reason in this moment a lidar survey is in progress.

So this technique seems to report an impressive precision and a very good accuracy, but it's still not evident if there are time and cost advantages comparing with more 'classical' methodologies; a weak point can be the need for a big number of GCPs, as an example in this test we used 21 differential GPS acquired points.

To evaluate the coherence of the obtained DSM with the actual objects present on the ground an high resolution orthophoto was realized showing another time a good fit (Fig.15). 


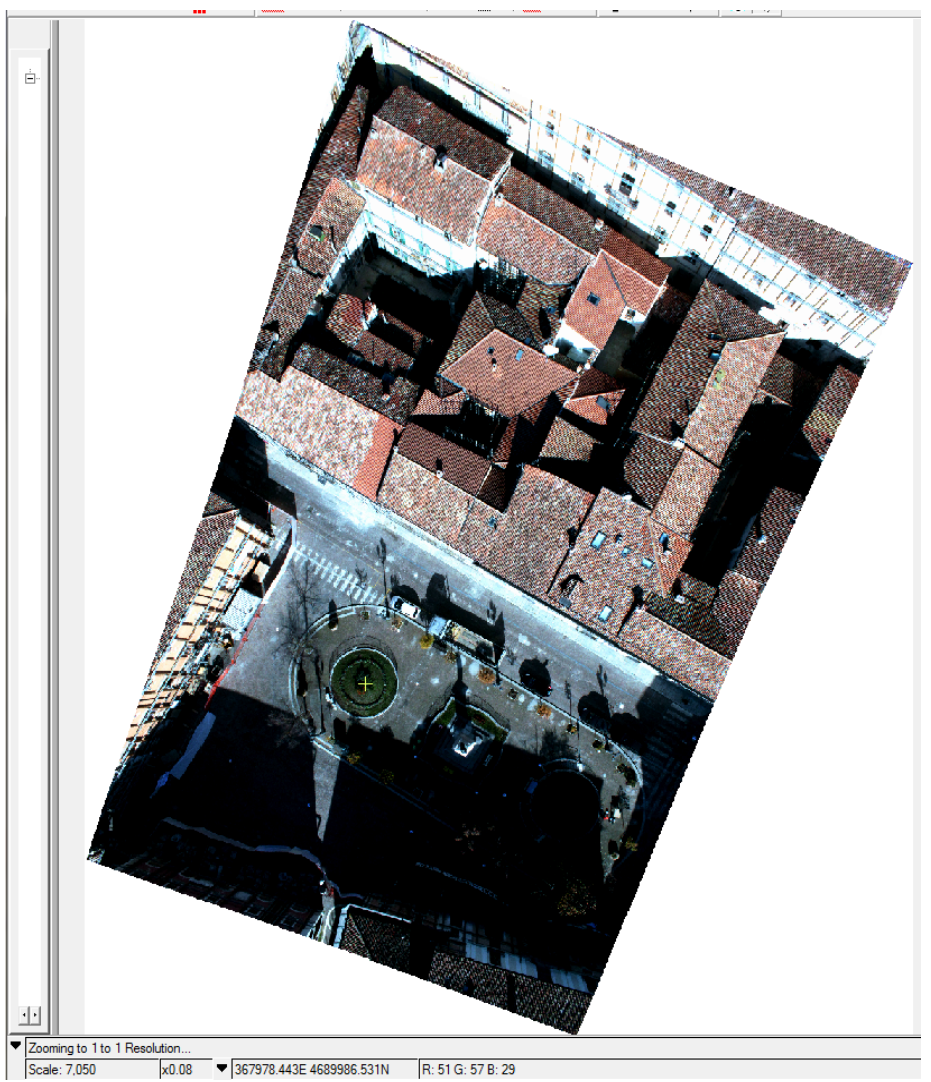

Fig. 15. Orthophoto resulting from processing

\section{Conclusion}

In this work, we wanted to show the advantages of the use of UAVs in the case of post-earthquake surveys scenarios, and how to optimize the flight planning can have an impact on the stereoscopic reconstruction of the area of investigation. Some first results confirmed that the geometric conditions hypothesized are correct to automatically extract DSM but further and more extensive test have to be performed. A lidar survey is in progress in this moment, and it will may possible to have a correct evaluation of the accuracy. The correct planning of the acquisitions is very important also considering the reduced autonomy of "Micro" UAVs. It has to be deeper investigated if a similar approach can be compatible with the instability observed in some UAV acquisitions. The tested algorithms will be also implemented in a graphical environment like the available open source GIS to facilitate the planning phase. 
Acknowledgements. The authors thank the IPT company of Rome, for having made available to Mikrokopter Okto platform in configuration, which made it possible to test.

\section{References}

1. Eynard, D., Vasseur, P., Demonceaux, C., Fremont, V.: UAV Altitude Estimation by Mixed Stereoscopic Vision. In: The IEEE/RSJ International Conference on Intelligent Robots and Systems, Taipei, Taiwan (2010)

2. Eisenbeiss, H., Haarbrink, R.B.: Accurate DSM production from unmanned helicopter systems. In: ISPRS Technical Commission VII Symposium 100 Years ISPRS Advancing Remote Sensing Science, Vienna, Austria (2010)

3. Tisdale, J., Kim, Z., Karl Hedrick, J.: Autonomous UAV Path Planning and Estimation. In: IEEE Robotics \& Automation Magazine. IEEE (2009), 1070-9932/09/2009

4. Hrabar, S.: 3D Path Planning and Stereo-based Obstacle Avoidance for Rotorcraft UAVs. In: 2008 IEEE/RSJ International Conference on Intelligent Robots and Systems Acropolis Convention Center Nice, France (2008)

5. Atkins, E.M., Millers, R.H., Pelts, T.V., Shaw, K.D., Ribbenss, W.B., Washabaugh, P.D.: Solus: An Autonomous Aircraft for Flight Trajectory Planning Research. In: American Control Conference Philadelphia, Pennsylvania (1998)

6. Nikolos, I.K., Valavanis, K.P., Tsourveloudis, N.C., Kostaras, A.N.: Evolutionary Algorithm Based Offline/Online Path Planner for UAV Navigation. IEEE Transactions on Systems, Man, and Cybernetics-Part B: Cybernetics 33(6) (2003)

7. Jun, M.: Path planning for unmanned aerial vehicles in uncertain and adversarial environments. In: Butenko, S., Murphey, R., Pardalos, P. (eds.) The Book Chapter of Cooperative Control: Models, Applications and Algorithms. Kluwer (2008)

8. Adolf, F.-M., Hirschmüller, H.: Meshing and Simplification of High Resolution Urban Surface Data for UAV Path Planning. J. Intell. Robot Syst. 61, 169-180 (2011), doi:10.1007/s10846-010-9478-8

9. Kraus, K.: Italian translation Prof. Sergio DEQUAL - Politecnico di Torino Cap 3, 4th edn., pp. 147-153 (1994)

10. Barazzetti, L., Nex, F., Remondino, F., Sarazzi, D., Scaioni, M.: UAV photogrammetry for mapping and 3D modeling - current status and future perspectives. In: International Archives of the Photogrammetry, Remote Sensing and Spatial Information Sciences, Conference on Unmanned Aerial Vehicle in Geomatics, Zurich, Switzerland, vol. XXXVIII-1/C22 UAV-g (2011)

11. Chiabrando, F., Nex, F., Piatti, D., Rinaudo, F.: UAV and RPV systems for photogrammetric surveys in archaelogical areas: two tests in the Piedmont region (Italy). Journal of Archaeological Science 38, 697-710 (2011)

12. Ercolin, L., Julitta, F., Montagna, M., Rigon, P., Sarazzi, D.: Moderne tecniche di rilievo aereo-fotogrammetrico di oggetti a sviluppo verticale per la produzione di DSM: il caso di Campione del Garda (BS). In: ASITA 2011 (2011)

13. Stefanik, K.V., Gassaway, J.C., Kochersberger, K., Lynn Abbott, A.: UAV-Based Stereo Vision for Rapid Aerial Terrain Mapping. GIScience \& Remote Sensing 48(1), 24-49 (2011), doi:10.2747/1548-1603.48.1.24 
14. Nebiker, S., Eugster, H., Flückiger, K., Christen, M.: Planning and management of realtime geospatial UAVs missions within a virtual globe environment. In: International Archives of the Photogrammetry, Remote Sensing and Spatial Information Sciences, Conference on Unmanned Aerial Vehicle in Geomatics, Zurich, Switzerland, vol. XXXVIII-1/C22 UAV-g (2011)

15. Eynard, D., Demonceaux, C., Vasseur, P., Fremont, V.: UAV Motion Estimation using Hybrid Stereoscopic Vision. In: MVA 2011 IAPR Conference on Machine Vision Applications, Nara, Japan (2011)

16. Neininger, B., Hacker, J.M.: Manned or unmanned - does this really matter? In: International Archives of the Photogrammetry, Remote Sensing and Spatial Information Sciences, Zurich, Switzerland. Conference on Unmanned Aerial Vehicle in Geomatics, vol. XXXVIII-1/C22 UAV-g (2011)

17. Moore, R.J.D., Thurrowgood, S., Bland, D., Soccol, D., Srinivasan, M.V.: UAV Altitude and Attitude Stabilisation using a Coaxial Stereo Vision System. In: 2010 IEEE International Conference on Robotics and Automation Anchorage Convention District, Anchorage, Alaska, USA (2010)

18. Eisenbeiss, H., Gaisecker, T., Hanusch, T., Kupferschmidt, D., Lambers, K., Sauerbier, M., Sotoodeh, S.: Combining photogrammetry and laser scanning for the recording and modelling of the Late Intermediate Period site of Pinchango Alto, Palpa, Peru. Journal of Archaeological Science 34, 1702-1712 (2007)

19. Richards, A., Bellingham, J., Tillerson, M., How, J.: Coordination and control of multiple UAVs. In: AIAA Guidance, Navigation, and Control Conference and Exhibit 2002, Monterey, California (2002) 